\title{
Peridinin, a carotenoid, inhibits proliferation and survival of HTLV-1-infected T-cell lines
}

\author{
CHIE ISHIKAWA $^{1,2}$, TAKAHIRO JOMORI ${ }^{3}$, JUNICHI TANAKA $^{3}$, MASACHIKA SENBA $^{4}$ and NAOKI MORI ${ }^{1}$ \\ ${ }^{1}$ Department of Microbiology and Oncology, Graduate School of Medicine, University of the Ryukyus, Nishihara, \\ Okinawa 903-0215; ${ }^{2}$ Division of Health Sciences, Transdisciplinary Research Organization for Subtropics and Island Studies, \\ ${ }^{3}$ Department of Chemistry, Biology and Marine Science, University of the Ryukyus, Nishihara, Okinawa 903-0213; \\ ${ }^{4}$ Department of Pathology, Institute of Tropical Medicine, Nagasaki University, Nagasaki 852-8523, Japan
}

Received May 13, 2016; Accepted July 1, 2016

DOI: $10.3892 /$ ijo.2016.3648

\begin{abstract}
Human T-cell leukemia virus type 1 (HTLV-1) causes either adult T-cell leukemia (ATL) or chronic inflammatory disorders such as HTLV-1-associated myelopathy/ tropical spastic paraparesis. These diseases are not curable as yet; therefore new agents for treatment and prevention are needed. Carotenoids are natural plant compounds with anti-carcinogenic activities. Peridinin is one of the most abundant carotenoids found in nature. Based on a series of past experiments, here we investigated the effects of peridinin extracted from Okinawan coral Isis hippuris on the proliferation and survival of HTLV-1-infected T-cell lines. The results of water-soluble tetrazolium-8 assay indicated that peridinin dose-dependently inhibits cell proliferation and viability of HTLV-1-infected T-cell lines. Flow cytometry showed that low concentration of peridinin induced cell cycle arrest at $G_{1}$ phase, while higher concentration induced apoptosis. Peridinin caused cleavage of caspase-3, -8 and -9. Peridinin significantly reduced the expression of $\mathrm{G}_{1}$ cell cycle regulators, including cyclin D1, cyclin D2, CDK4, CDK6 and c-Myc, and anti-apoptotic proteins, including survivin, XIAP and $\mathrm{Bcl}-2$, in a dose-dependent manner. Peridinin suppressed DNA binding of NF- $\kappa$ B. Peridinin inhibited phosphorylation of I $\kappa \mathrm{B} \alpha$, RelA, Akt and p70 S6 kinase, and reduced protein expression level of 3-phosphoinositide-dependent protein kinase 1. Thus, peridinin exerts its anti-proliferative and pro-apoptotic effects by suppressing NF- $\mathrm{BB}$ and Akt signaling in HTLV-1-infected T cells. Peridinin also reduced tumor growth in mice harboring ATL xenograft tumors. The results suggested that peridinin is a potentially suitable therapeutic agent against HTLV-1associated diseases.
\end{abstract}

Correspondence to: Professor Naoki Mori, Department of Microbiology and Oncology, Graduate School of Medicine, University of the Ryukyus, 207 Uehara, Nishihara, Okinawa 903-0215, Japan

E-mail: naokimori50@gmail.com

Key words: peridinin, adult T-cell leukemia, human T-cell leukemia virus type 1 , nuclear factor- $\kappa \mathrm{B}, \mathrm{Akt}$

\section{Introduction}

Human T-cell leukemia virus type 1 (HTLV-1) is etiologically associated with two major diseases, adult T-cell leukemia (ATL) and HTLV-1-associated myelopathy/tropical spastic paraparesis (HAM/TSP) (1). ATL is an aggressive malignant disease of $\mathrm{CD}^{+} \mathrm{T}$ cells that is highly resistant to currently available chemotherapies, and HAM/TSP presents with inflammatory symptoms and incomplete paralysis of the limbs. Although various features of HTLV-1 biology have been defined, the treatment of the aggressive subtypes of ATL and HAM/TSP remains inadequate with minimal improvements.

Carotenoids have numerous bioactivities. In particular, the marine carotenoid, fucoxanthin, and its metabolite, fucoxanthinol, have multiple functions and studies from our laboratories have shown that both carotenoids have considerable potential for preventions and treatment of cancer (2-5). In contrast, the bioactivities of peridinin, a carotenoid with a structure similar to that of fucoxanthin (Fig. 1), have not been well studied. Peridinin is cytotoxic to colorectal cancer cells in vitro (6). However, so far, there is no report on the use of peridinin in HTLV-1-associated diseases including ATL. In this study, we tested the anti-ATL activity of peridinin isolated from the gorgonian Isis hippuris both in vitro and in vivo, and determined the underlying molecular mechanisms of its anti-ATL effects. The results showed the sensitivity of HTLV-1-infected T-cell lines to peridinin and that peridinin is a potentially promising drug for HTLV-1-associated diseases.

\section{Materials and methods}

Cell culture. HTLV-1-transformed T-cell lines, MT-2, MT-4, HUT-102, C5/MJ and SLB-1, and ATL-derived T-cell lines, MT-1 and ED-40515(-), were maintained in RPMI-1640 medium (cat. no. 30264-56, Nacalai Tesque, Inc., Kyoto, Japan) supplemented with $10 \%$ fetal bovine serum (Biological Industries, Kibbutz Beit Haemek, Israel) and 1\% penicillin/ streptomycin (cat. no. 09367-34, Nacalai Tesque, Inc.)

Purification of peridinin. A hexane extract (totally $36.2 \mathrm{~g}$ ) of the gorgonian I. hippuris, collected July 1980, was subjected to separation first on a silica gel column and then 
on Sephadex LH20 (cat. no. 17-0090-03, Pharmacia, Uppsala, Sweden) column to give a crude peridinin fraction (591 mg), which was kept frozen for $>30$ years. Then, the fraction was passed through a Sephadex LH20 column (MeOH- $\mathrm{CH}_{2} \mathrm{Cl}_{2}$, 1-1) twice followed by silica (silica gel 60; cat. no. 107734 , Merck, Darmstadt, Germany) flash column and silica HPLC (cat. no. 38005-51, Cosmosil 5SL-II, 10x250 mm; Nacalai Tesque, Inc.) (hexane-EtOAc, 1-1) to yield $9.0 \mathrm{mg}$ of peridinin. Additional amount of peridinin was purified from crude fractions of another specimen of the same gorgonian, with a final total yield of $20.0 \mathrm{mg}$ of peridinin. The ${ }^{1} \mathrm{H}$ NMR spectrum of the isolated peridinin in $\mathrm{CDCl}_{3}$ (cat. no. 139-18001, Wako Pure Chemical Industries, Osaka, Japan) was identical to that reported previously (7).

Measurement of cell proliferation and cytotoxicity. Cell proliferation and cytotoxicity were measured by the water-soluble tetrazolium (WST)-8 assay (cat. no. 07553-44, Nacalai Tesque, Inc.). The WST- 8 is taken up by viable cells and reduced to the colored formazan product by mitochondrial dehydrogenase (8). For the WST-8 assay, $100 \mu 1\left(1 \times 10^{4}\right)$ cells were seeded in 96-well plates and treated with different concentrations of peridinin or dimethyl sulfoxide (DMSO) (cat. no. 13407-45, Nacalai Tesque, Inc.) for $24 \mathrm{~h}$. Then, $10 \mu \mathrm{l}$ of WST- 8 was added to each well and incubated for $6 \mathrm{~h}$. Absorbance was measured at 450 and $620 \mathrm{~nm}$ by an iMark ${ }^{\mathrm{TM}}$ microplate absorbance reader (Bio-Rad Laboratories, Inc., Hercules, CA, USA). Cell viability was calculated by the percentage of treated cells relative to that of solvent controls. All experiments were performed in triplicates.

Cell cycle analysis. The cells were treated with different concentrations of peridinin or DMSO for $24 \mathrm{~h}$ and stained with the CycleTEST Plus DNA Reagent kit (cat. no. 340242, Becton-Dickinson Immunocytometry Systems, San Jose, CA, USA) for analysis of changes in the cell cycle. The stained cells were analyzed by an Epics XL flow cytometer (Beckman Coulter, Inc., Brea, CA, USA). The MultiCycle software (version 3.0) was used to calculate the percentage of cells in each cell cycle phase.

Apoptosis assay. Apoptosis was assessed by the APO2.7 assay. Cells were seeded in culture plates then treated with different concentrations of peridinin or DMSO for $24 \mathrm{~h}$, followed by analysis by flow cytometry after staining with phycoerythrinconjugated APO2.7 antibody (cat. no. IM2088, Beckman Coulter, Marseille, France), which specifically detects 7A6, a 38-kDa mitochondrial membrane antigen expressed during apoptosis (9). For analysis of morphological changes in nuclei, cells stained with DNA-specific Hoechst 33342 dye (cat. no. 346-07951, Dojindo Molecular Technologies, Inc., Kumamoto, Japan) were analyzed using a Leica DMI6000 microscope (Leica Microsystems, Wetzlar, Germany). In addition, apoptosis was also assessed by monitoring cleavage of caspase-3, -8 and -9, as well as poly(ADP-ribose) polymerase (PARP) by western blot analysis.

Protein extraction and western blotting. Cells were harvested after treatment and lysed in a lysis buffer containing $62.5 \mathrm{mM}$ Tris-HCl (pH 6.8) (cat. no. 35434-21, Nacalai Tesque, Inc.),
$2 \%$ sodium dodecyl sulfate (cat. no. 31607-65, Nacalai Tesque, Inc.), 10\% glycerol (cat. no. 17045-65, Nacalai Tesque, Inc.), 6\% 2-mercaptoethanol (cat. no. 21438-82, Nacalai Tesque, Inc.) and $0.01 \%$ bromophenol blue (cat. no. 021-02911, Wako Pure Chemical Industries). The lysates were centrifuged and the supernatants were collected. The cell lysates $(20 \mu \mathrm{g})$ were separated on sodium dodecyl sulfate-polyacrylamide gels followed by electroblotting to polyvinylidene difluoride membranes (cat. no. IPVH00010EMD, Millipore, Darmstadt, Germany). The following antibodies were used: cleaved caspase-3 (cat. no. 9664), -8 (cat. no. 9496) and -9 (cat. no. 9501), cleaved PARP (cat. no. 9541), survivin (cat. no. 2808), Bak (cat. no. 3814), Bcl-xL (cat. no. 2762), Akt (cat. no. 9272), phospho-Akt (Ser473) (cat. no. 4060), phospho-Akt (Thr308) (cat. no. 13038), phospho-IкB $\alpha$ (Ser32 and Ser36) (cat. no. 9246), phospho-IкB kinase (IKK) $\alpha / \beta$ (Ser176 and Ser180) (cat. no. 2694), RelA (cat. no. 8242), phospho-RelA (Ser536) (cat. no. 3033), 3-phosphoinositide-dependent protein kinase 1 (PDK1) (cat. no. 3062), phospho-PDK1 (Ser241) (cat. no. 3061), phospho-p70 S6 kinase (S6K) (Thr421 and Ser424) (cat. no. 9204), phospho-phosphatase and tensin homologue (PTEN) (Ser380) (cat. no. 9551) and phosphoextracellularly regulated kinase (Erk)1/2 (Thr202 and Tyr204) (cat. no. 4370) were all from Cell Signaling Technology, Inc. (Beverly, MA, USA); c-IAP2 (cat. no. sc-7944), cyclin D2 (cat. no. sc-593) and $\mathrm{I} \kappa \mathrm{B} \alpha$ (cat. no. sc-371) were from Santa Cruz Biotechnology, Inc. (Santa Cruz, CA, USA); XIAP (cat. no. M044-3) and cyclin D1 (cat. no. K0062-3) were from Medical \& Biological Laboratories, Co. (Aichi, Japan); Bcl-2 (cat. no. MS-597), CDK4 (cat. no. MS-299), CDK6 (cat. no. MS-398), c-Myc (cat. no. MS-1054) and actin (cat. no. MS-1295) were from Neomarkers, Inc. (Fremont, CA, USA).

Preparation of nuclear extracts and electrophoretic mobility shift assay (EMSA). Nuclear proteins were extracted and transcription factors bound to specific DNA sequences were examined by EMSA, as described previously (10). The top strand sequences of the oligonucleotide probe or competitors were as follows: for the nuclear factor- $\kappa \mathrm{B}(\mathrm{NF}-\kappa \mathrm{B})$ element of the interleukin-2 receptor $\alpha$ chain $(I L-2 R \alpha)$ gene, 5'-GATCCGGCAGGGGAATCTCCCTCTC-3'; and for the activator protein-1 (AP-1) element of the interleukin-8 (IL-8) gene, 5'-GATCGTGATGACTCAGGTT-3'. The above underlined sequences are the $\mathrm{NF}-\kappa \mathrm{B}$ and AP-1 binding sites, respectively. In competition experiments, the nuclear extracts were preincubated with 100 -fold excess of unlabeled oligonucleotides for $15 \mathrm{~min}$. To identify $\mathrm{NF}-\kappa \mathrm{B}$ proteins in the DNA-protein complex shown by EMSA, antibodies specific for various $\mathrm{NF}-\kappa \mathrm{B}$ family proteins, including p50 (cat. no. sc-114X), RelA (cat. no. sc-109X), c-Rel (cat. no. sc-70X), p52 (cat. no. sc-298X) and RelB (cat. no. 226X) (Santa Cruz Biotechnology Inc.) were used. These antibodies were incubated with the nuclear extracts for $45 \mathrm{~min}$ at room temperature before incubation with the radiolabeled probe.

Xenograft tumor model. Five-week-old female C.B-17/ Icr-severe combined immune deficiency (SCID) mice were obtained from Kyudo, Co. (Tosu, Japan). The mice were kept in specific pathogen-free conditions and housed in cages main- 


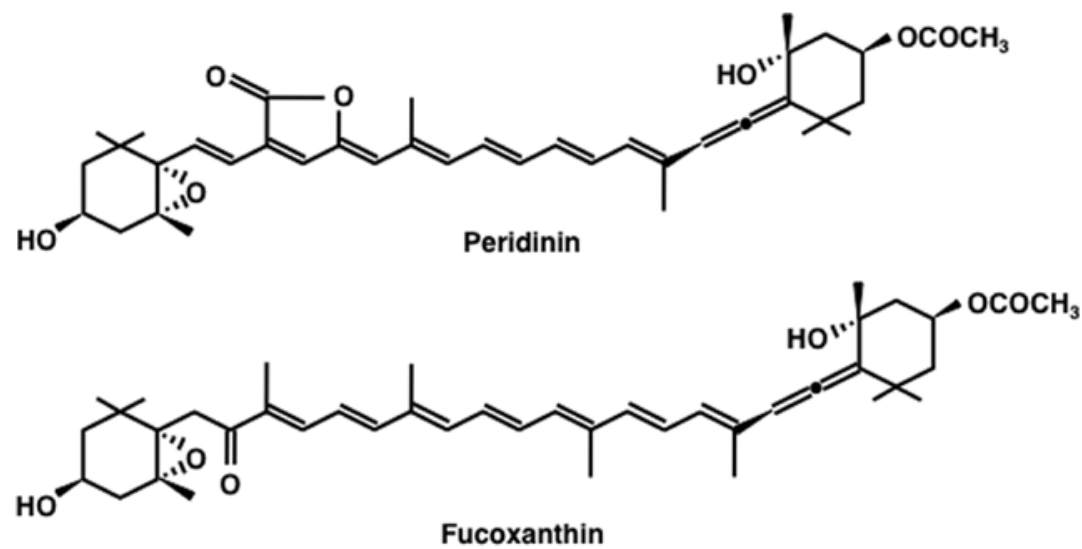

Figure 1. Chemical structures of peridinin and fucoxanthin.

tained in air-conditioned rooms (temperature, $24^{\circ} \mathrm{C}$; humidity, $60 \%$ ) set at 12-h light/12-h dark cycles. Mice were provided with standard rodent diet (CE-2 from CLEA Japan, Inc., Tokyo, Japan) and water ad libitum. To induce malignancy, $1 \times 10^{7}$ HUT-102 cells suspended in $300 \mu$ l sterile RPMI-1640 medium were inoculated subcutaneously into the postauricular region of the SCID mice, which were then divided randomly into two treatment groups ( $\mathrm{n}=6 /$ group). Peridinin was solubilized in 5.2\% polyethylene glycol 400 (cat. no. 161-09065, Wako Pure Chemical Industries) and 5.2\% Tween-80 (cat. no. 231181, Becton-Dickinson, Franklin Lakes, NJ, USA) at a concentration of $0.56 \mathrm{mg} / \mathrm{ml}$, and administered intraperitoneally for 5 days/week with a 2-day rest, and the treatment was continued for 21 days, beginning on the day subsequent to cell inoculation. The control group received vehicle only, while the treated group received peridinin at dose of $8.5 \mathrm{mg} / \mathrm{kg}$. Tumor diameter was measured weekly with a shifting caliper, and tumor volume was calculated. Mice were weighed weekly. All mice were sacrificed on day 21 when tumors did not reach the ethically allowed maximal size. Subsequently, tumors were excised and their weight was measured. This study was performed according to the Guidelines for Animal Experimentation of the University of the Ryukyus (Nishihara, Japan), and was approved by the Animal Care and Use Committee of the University of the Ryukyus (reference no. 6042).

Morphological analysis of tumor tissues and terminal deoxynucleotidyl transferase deoxyuridine triphosphate nick end labeling (TUNEL) assay. Tumor specimens were collected from the control and peridinin-treated groups, fixed in formalin (Wako Pure Chemical Industries) solution, dehydrated through graded ethanol series (Japan Alcohol Selling Co., Tokyo, Japan) and embedded in paraffin (cat. no. 09620, Sakura Finetek Japan Co., Tokyo, Japan). The paraffin-embedded specimens of ATL tumors were stained with hematoxylin and eosin (H\&E; cat. nos. 234-12 and 1159350025, Merck) and examined histologically. Analysis of DNA fragmentation by TUNEL assay was performed using a commercial kit (cat. no. 11684817910, Roche Applied Science, Penzberg, Germany). Cells were examined under a light microscope (Axioskop 2 Plus) with an Achroplan 40x/0.65 lens (both from Zeiss, Hallbergmoos, Germany). Images were acquired with an AxioCam 503 color and AxioVision LE64 software (Zeiss).

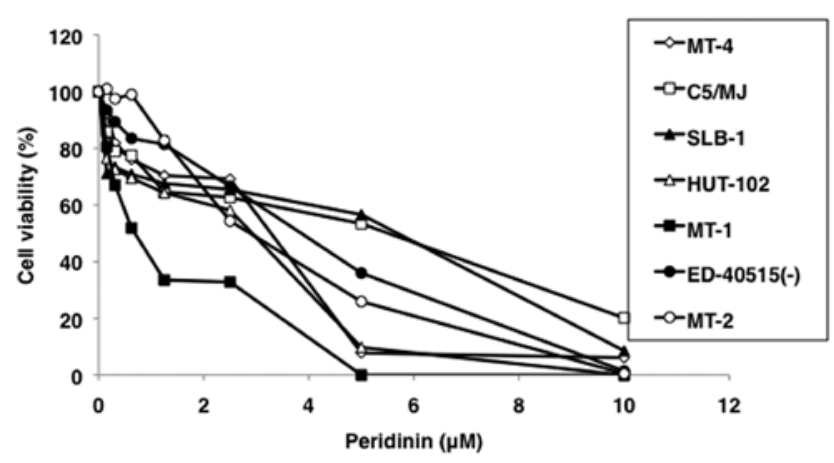

Figure 2. Peridinin inhibits cell viability of various HTLV-1-infected T-cell lines. Several types of HTLV-1-infected T-cell lines were treated with various concentrations of peridinin for $24 \mathrm{~h}$ followed by WST- 8 assay $(n=3)$. The results represent cell viability of peridinin-treated cells relative to that of control cells (cells that were left untreated).

Biomarker analysis. Serum concentrations of human soluble IL-2R (sIL-2R; cat. no. DR2A00, R\&D Systems, Inc., Minneapolis, MN, USA) and human soluble cluster of differentiation 30 (sCD30; cat. no. RBMS240R, BioVendor Inc., Brno, Czech Republic) were measured by enzyme-linked immunosorbent assay (ELISA), according to the protocol supplied by the manufacturer.

Statistical analysis. All values are expressed as the mean \pm standard deviation (SD). Differences between the control and treated groups were tested for statistical significance by the unpaired t-test. A P-value $<0.05$ denoted the presence of statistically significant difference.

\section{Results}

Peridinin suppresses the survival of HTLV-1-infected T-cell lines. The suppressive effect of peridinin on the survival of HTLV-1-transformed T-cell lines MT-2, MT-4, HUT-102, C5/MJ, SLB-1, and patient-derived ATL cell lines MT-1 and ED-40515(-) was confirmed by WST-8 assay. Furthermore, peridinin dose-dependently suppressed the viability of HTLV-1-infected T-cell lines (Fig. 2). The $\mathrm{IC}_{50}$ values of peridinin on HTLV-1-infected T-cell lines as estimated by the WST-8 assay were $0.71-5.38 \mu \mathrm{M}$ (Table I). 
Table I. The $\mathrm{IC}_{50}$ values for HTLV-1-infected T-cell lines.

\begin{tabular}{llc}
\hline & \multicolumn{1}{c}{ Cell line } & $\mathrm{IC}_{50}(\mu \mathrm{M})$ \\
\hline HTLV-1-transformed & MT-2 & 2.23 \\
HTLV-1-transformed & MT-4 & 3.06 \\
HTLV-1-transformed & C5/MJ & 5.15 \\
HTLV-1-transformed & SLB-1 & 5.38 \\
HTLV-1-transformed & HUT-102 & 2.76 \\
ATL-derived & MT-1 & 0.71 \\
ATL-derived & ED-40515(-) & 3.53 \\
\hline
\end{tabular}

Effects of peridinin on cell cycle distribution. Disturbance of the cell cycle is one of therapeutic targets for development of new anticancer drugs. The MT-2 and HUT-102 cell lines were treated with different concentrations of peridinin for $24 \mathrm{~h}$ and cell cycle was analyzed by propidium iodide staining and DNA content analysis by flow cytometry. Low-dose peridinin $(1.25,2.5$ and $5.0 \mu \mathrm{M})$ significantly reduced the proportion of cells in the $S$ phase but increased those of cells in the $G_{1}$ phase population (Fig. 3A and B). These results suggest that peridinin causes $\mathrm{G}_{1}$ cell cycle arrest in HTLV-1-infected T-cell lines. The increase in $\mathrm{G}_{1}$ cell cycle arrest was peridinin dose- and time-dependent (Fig. 3). On the other hand, the percentages of cells in the sub- $\mathrm{G}_{1}$ phase of the high-dose group $(10 \mu \mathrm{M})$ (MT-2, 28.4\%; HUT-102, 21.3\%) were significantly higher than those of low-dose groups and control (MT-2, 3.1\%; HUT-102, $6.9 \%$ ) at $24 \mathrm{~h}$ (Fig. 3A). Furthermore, peridinin at high dose markedly increased the percentage of apoptotic cells of sub- $\mathrm{G}_{1}$ population and this effect was time-dependent (Fig. 3C).

Effects of peridinin on apoptosis of HTLV-1-infected T-cell lines. MT-2 and HUT-102 cells were treated with different concentrations of peridinin for $24 \mathrm{~h}$ and apoptosis was assessed by APO2.7 staining. There was no significant difference in rates of apoptosis between the low-dose groups (1.25, 2.5 and $5.0 \mu \mathrm{M})$ and negative control at $24 \mathrm{~h}$, while high-dose peridinin $(10 \mu \mathrm{M})$ induced significant apoptosis of MT-2 and HUT-102 cells (Fig. 4A). As shown in Fig. 4B, by Hoechststained MT-2 cells showed distinct morphological features of chromatin condensation and fragmented nuclei in the presence of $10 \mu \mathrm{M}$ peridinin. In another method for apoptosis based on caspase-3, -8, -9 and PARP, increases in cleaved caspase-3, $-8,-9$ and PARP expression levels were detected in MT-2 cells treated with $10 \mu \mathrm{M}$ peridinin (Fig. 4C).

Apoptosis is regulated by a balance between pro-apoptotic and anti-apoptotic proteins. The Bcl-2 family members play major roles in cell survival and apoptosis (11). For this reason,
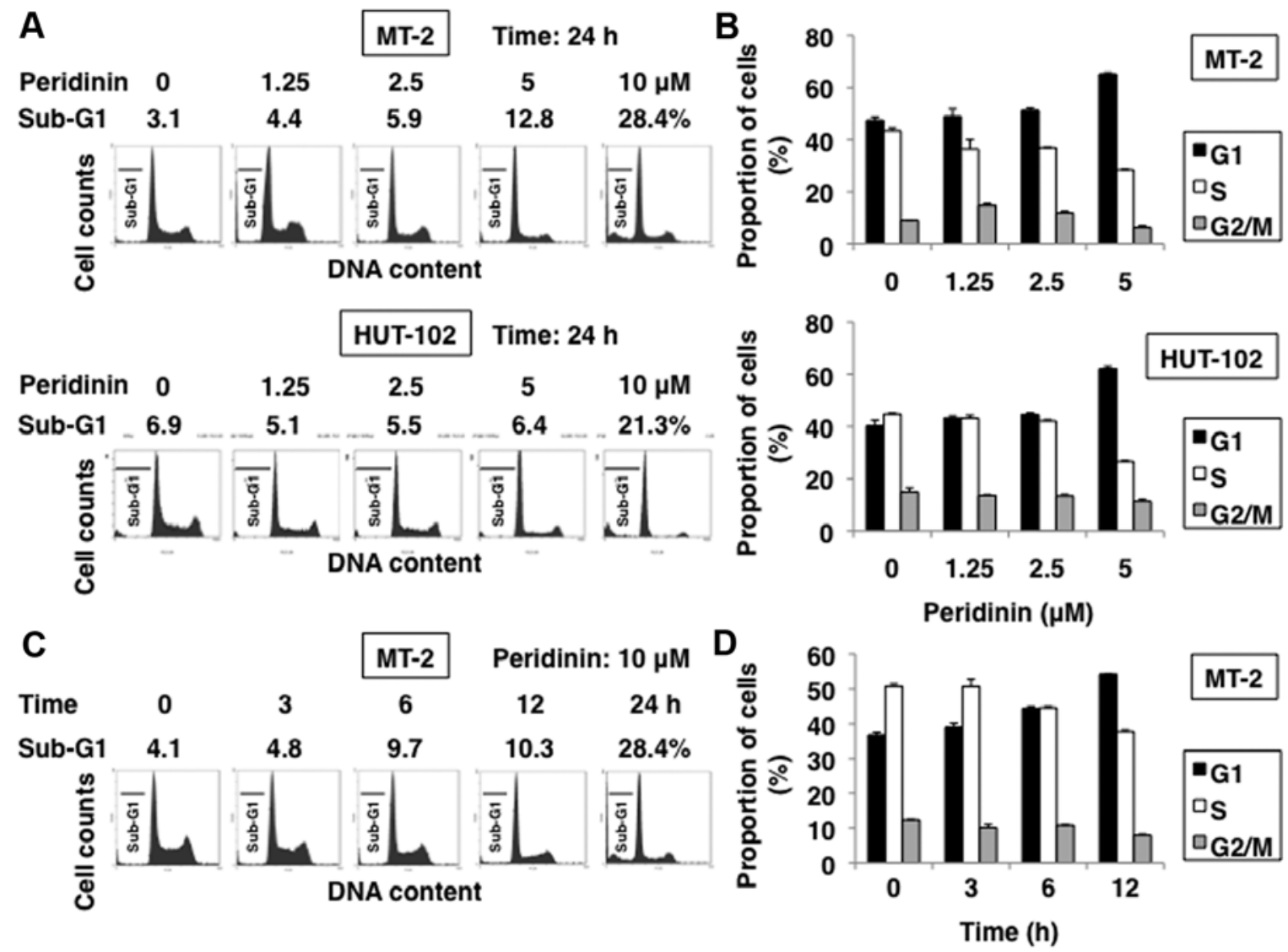

Figure 3. Effects of peridinin on the cell cycle distribution of HTLV-1-infected T-cell lines. MT-2 and HUT-102 cells were treated with the indicated concentrations of peridinin, harvested after the indicated time-points and then labeled. The labeled cells were analyzed using flow cytometry and the percentage of cells in each cell cycle phase was calculated with software ( $n=3)$. (A) Percentage of cells in the sub- $\mathrm{G}_{1}$ phase of the high-dose group was higher than that of the low-dose groups and control. (B) Quantification of the flow cytometry histograms up to a concentration of $5 \mu \mathrm{M}$. Data are mean \pm SD. (C) Peridinin at high concentration increased the percentage of sub- $\mathrm{G}_{1}$ cells population in a time-dependent manner. (D) Quantification of the flow cytometry histograms up to a culture period of $12 \mathrm{~h}$. Data are mean $\pm \mathrm{SD}$. 

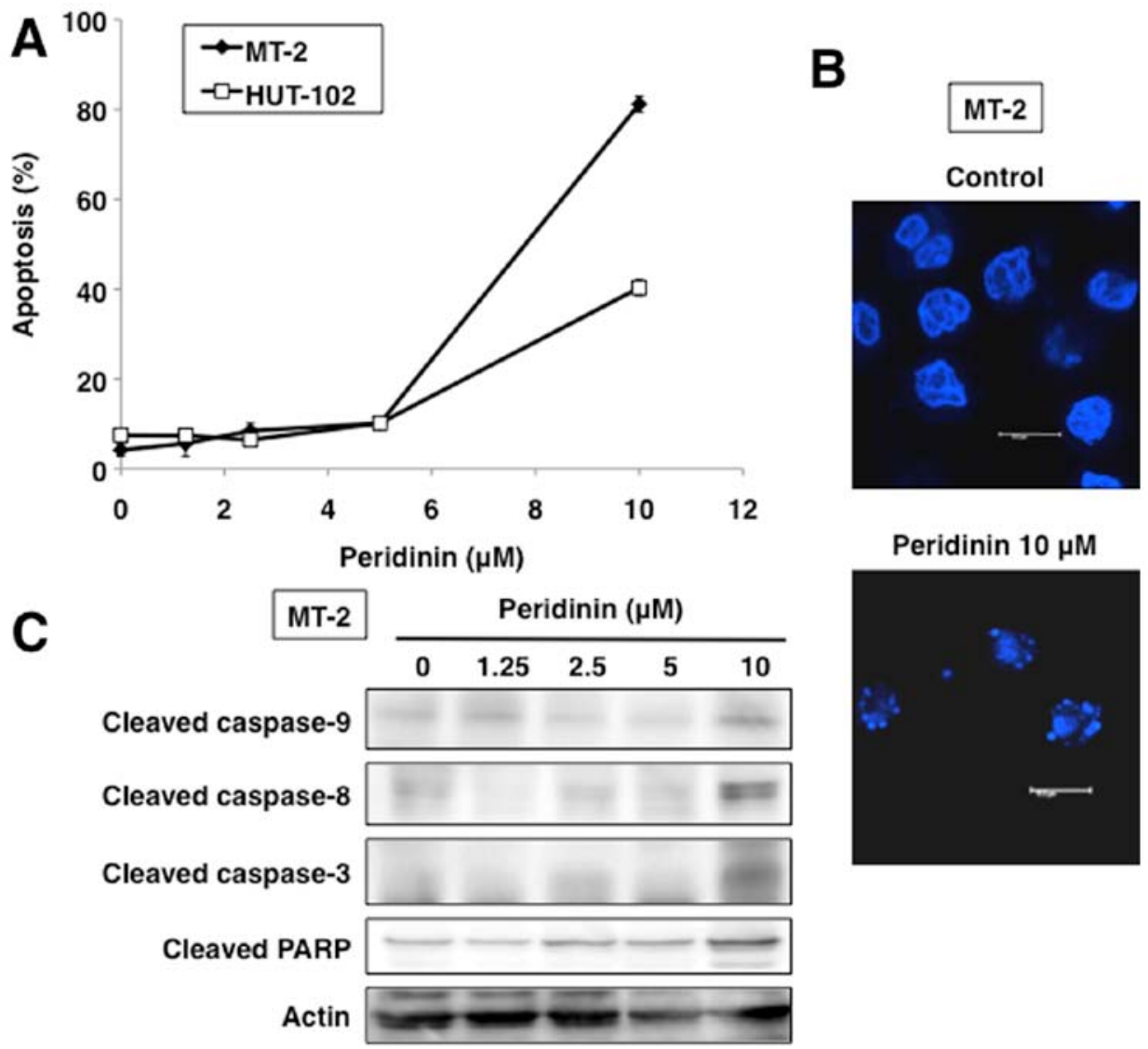

Peridinin $10 \mu \mathrm{M}$

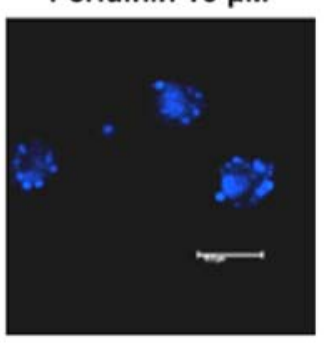

Figure 4. Peridinin induces apoptosis of HTLV-1-infected T-cell lines. (A) MT-2 and HUT-102 cells were treated with the indicated concentrations of peridinin for $24 \mathrm{~h}$. Cells were stained with APO2.7 antibody and then analyzed by flow cytometry. Each data point represents the mean \pm SD ( $\mathrm{n}=3$ ). (B) Morphological features of cells stained with Hoechst 33342. MT-2 cells were treated or untreated with $10 \mu \mathrm{M}$ peridinin for $24 \mathrm{~h}$. (C) Expression levels of cleaved caspase-3, $-8,-9$ and PARP in peridinin-treated MT-2 cells. MT-2 cells were treated with peridinin at the indicated concentrations for 24 h. After the treatment, the cells were collected, and then proteins were extracted and analyzed by western blot analysis.

we measured the effects of peridinin on the expression of Bcl-2, Bcl-xL and Bak by western blot analysis. Peridinin inhibited the expression of Bcl-2 in a dose-dependent manner, but not that of Bcl-xL and Bak. Inhibitor of apoptosis (IAP) proteins, including XIAP, c-IAP2 and survivin directly bind to activated caspase- $3,-7$ and -9 , and inhibit their activities (12). As shown in Fig. 5, peridinin reduced the protein levels of survivin and XIAP, but not c-IAP2, in cultured MT-2 cells and the effect was dose-dependent. These results suggest that peridinin inhibits cell survival and induces apoptosis through the regulation of Bcl-2 family members and IAPs.

Cell cycle progression is governed by a family of cyclins and CDKs, and cyclin D/CDK4/CDK6 is a critical determinant of progression through $\mathrm{G}_{1}$ phase of the cell cycle (13). We next evaluated the effects of peridinin on cyclins and CDKs involved in cell cycle arrest in MT-2 cells. As shown in Fig. 5, peridinin significantly decreased cyclin D1, cyclin D2, CDK4 and CDK6 levels in MT-2 cells.

Proto-oncogene $c$-myc encodes a basic loop-helix-loop zipper transcription factor that plays crucial roles in cell proliferation, apoptosis, differentiation and metabolism $(14,15)$. c-Myc can activate the expression of many downstream cell cycle regulators, such as cyclin D1, cyclin D2 and CDK4 (14-16). Peridinin downregulated c-Myc protein levels in MT-2 cells (Fig. 5), suggesting that c-Myc served as an upstream target in the peridinin-mediated blockade of cell cycle progression.

Peridinin inhibits $N F-\kappa B$. NF- $\kappa \mathrm{B}$ encompases a family of transcription factors that are involved in numerous biological processes including cell growth and survival. The $\mathrm{NF}-\kappa \mathrm{B}$ proteins are usually sequestered in the cytoplasm by a family of inhibitors including $\mathrm{I} \kappa \mathrm{B} \alpha$. Activation of NF- $\mathrm{NB}$ occurs via phosphorylation of $I \kappa B \alpha$ at Ser32 and Ser36. This is followed by proteasome-mediated degradation resulting in release and nuclear translocation of active $\mathrm{NF}-\kappa \mathrm{B}$, where it regulates the expression of several pro-survival proteins and cell cycle regulatory molecules (17). Interestingly, many genes downregulated by peridinin (cyclin D1, cyclin D2, CDK4, CDK6, c-Myc, Bcl-2, XIAP and survivin) are also regulated by NF- $\kappa \mathrm{B}(18-25)$. Culture of MT-2 cells in the presence of peridinin resulted in a significant dose-dependent inhibition of $\mathrm{I} \kappa \mathrm{B} \alpha$ protein phosphorylation (Fig. 6A). Pursuing further this line of experiments, we examined, using the EMSA, the binding of $\mathrm{NF}-\kappa \mathrm{B}$ family proteins to the $\mathrm{NF}-\kappa \mathrm{B}$ sequence of the $I L-2 R \alpha$ gene. To validate the relevance of the visualized bands with regard to the presence and specificity of the NF- $\mathrm{B}-\mathrm{DNA}$ complex, we incubated nuclear extracts from MT-2 cells with antibodies specific for $N F-\kappa B$ family proteins as well as to an excess of unlabeled competitors $N F-\kappa B$ and 


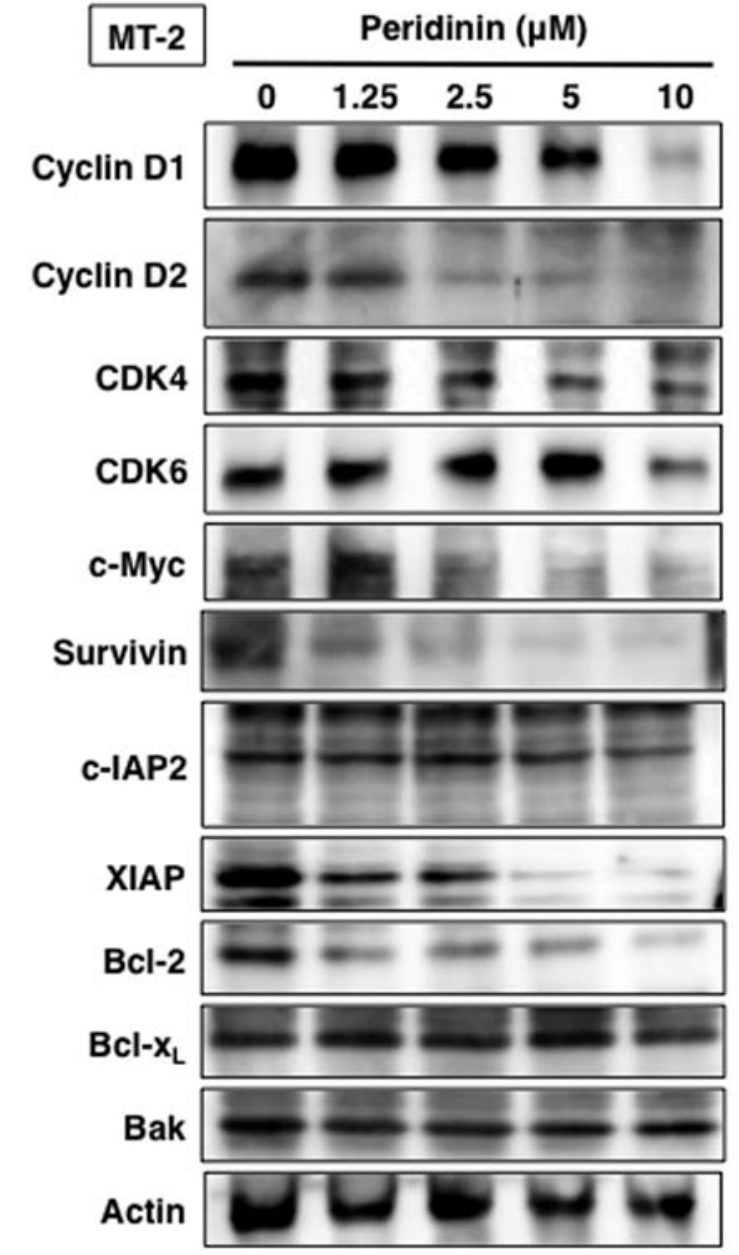

Figure 5. Expression levels of cell cycle- and apoptosis-related proteins after treatment with various concentrations of peridinin. MT-2 cells were treated with the indicated concentrations of peridinin for $24 \mathrm{~h}$. Whole-cell extracts were prepared, and the lysate was analyzed by western blot analysis using the indicated antibodies.

AP-1 sequences. The band completely disappeared by excess unlabeled NF- $\mathrm{kB}$ sequence, but not by AP-1 sequence, and was affected by preincubation with antibodies specific for $\mathrm{p} 50$, RelA, c-Rel, p52 and RelB, indicating that this band contains the specific DNA-NF- $\kappa$ B subunit complex (Fig. 6B, right panel). Treatment with peridinin completely abolished this binding process (Fig. 6B, left panel), indicating that peridinin inhibits the NF- $\mathrm{KB}$ pathway. This inhibition can result from upstream signaling events or from direct inhibition of one or more stages of the NF- $\mathrm{BB}$ pathway. This latter possibility was assessed by determining the types of proteins along the $\mathrm{NF}-\kappa \mathrm{B}$ pathway that are direct targets for peridinin.

Attenuation of phosphorylation of IкB $\alpha$ (Fig. 6A) suggested that the upstream kinase IKK $\beta$ was the likely site of the inhibitory action of peridinin. To further explore this tenable conclusion, we examined IKK $\beta$ phosphorylation. The results showed that peridinin suppressed the phosphorylation of IKK $\beta$ (Fig. 6A).

Peridinin causes inhibition of phosphorylation of Akt protein. RelA is phosphorylated at Ser536 by a variety of kinases through various signaling pathways and such phosphorylation enhances RelA transactivation potential (26). As shown in Fig. 6A, peridinin significantly inhibited phosphorylation of RelA in MT-2 cells. Akt is a component of an essential pathway for cell survival and growth during carcinogenesis, and it can also phosphorylate RelA at Ser536 through an IKK $\alpha$ - or IKK $\beta$ dependent mechanism (26). Peridinin induced dose-dependent inhibition of Akt phosphorylation at Ser473 and Thr308 in MT-2 cells (Fig. 6C). Furthermore, peridinin suppressed the phosphorylation of IKK $\alpha$ and IKK $\beta$ (Fig. 6A). Considered together, the above results suggest that peridinin inhibits RelA phosphorylation by inhibiting Akt and IKK activation.

Peridinin inhibits PDK1 protein expression. PDK1 is an immediate downstream mediator of phosphoinositide 3-kinase (PI3K) and is activated upon phosphorylation at Ser241 (27). Activated PDK1 controls cell proliferation and survival by further activating its downstream cAMP-dependent, cGMPdependent, protein kinase $\mathrm{C}$ family of protein kinases, including Akt (28) and S6K (29). We next examined the effect of peridinin on PDK1 protein. Peridinin reduced phosphorylation and protein expression of PDK1 (Fig. 6C) and induced significant inhibition of S6K phosphorylation.

The tumor suppressor PTEN directly dephosphorylates phosphatidylinositol 3,4,5-triphosphate (PIP3) to PI(4,5)P2 and opposes the PI3K/Akt signaling (30). The oncogenicity of abnormal PI3K/Akt signaling is emphasized by the observation that deactivating mutations of the gene encoding PTEN are among the most frequently occurring in human malignancy (30). However, ATL cells do not harbor genetic alterations in PTEN but express high levels of PTEN that is highly phosphorylated through loss of tumor suppressor N-myc downstream-regulated gene 2 (31). It is believed that phosphorylation keeps PTEN in an inactive form in the cytoplasm (32). To evaluate whether peridinin increases PTEN activity by dephosphorylation, the phosphorylation status of PTEN was examined. However, peridinin did not affect PTEN phosphorylation (Fig. 6C). Erk pathway is also involved in HTLV-1 transforming protein Tax-mediated apoptosis protection (33), but peridinin did not inhibit Erk phosphorylation (Fig. 6C).

Peridinin inhibits tumorigenesis in vivo. To determine the antitumor activity of peridinin in vivo, HUT-102 cells were subcutaneously injected into the postauricular region of the SCID mice. Mice were intraperitoneally injected with vehicle or peridinin at $8.5 \mathrm{mg} / \mathrm{kg} 5$ days a week over a period of 21 days. Tumorigenesis was noted in all mice transplanted with HUT-102 cells (Fig. 7A), but none showed signs of discomfort and/or suffering until euthanasia. Mice treated with peridinin showed significantly inhibited tumor growth compared with the control group $(\mathrm{P}<0.005)$. Furthermore, the weight of the excised tumors was lower after treatment with peridinin compared with those of untreated mice, albeit statistically insignificant (Fig. 7B and C). In addition, mice seemed to tolerate treatment with peridinin without overt signs of toxicity or significant loss of body weight, similar to the vehicle-treated group (Fig. 7D).

Tumor sections from the treated mice were further examined by H\&E staining. Apoptosis of tumor cells was noted in the peridinin-treated group, which was characterized by cytoplasmic condensation, chromatin hyperchromatism and 


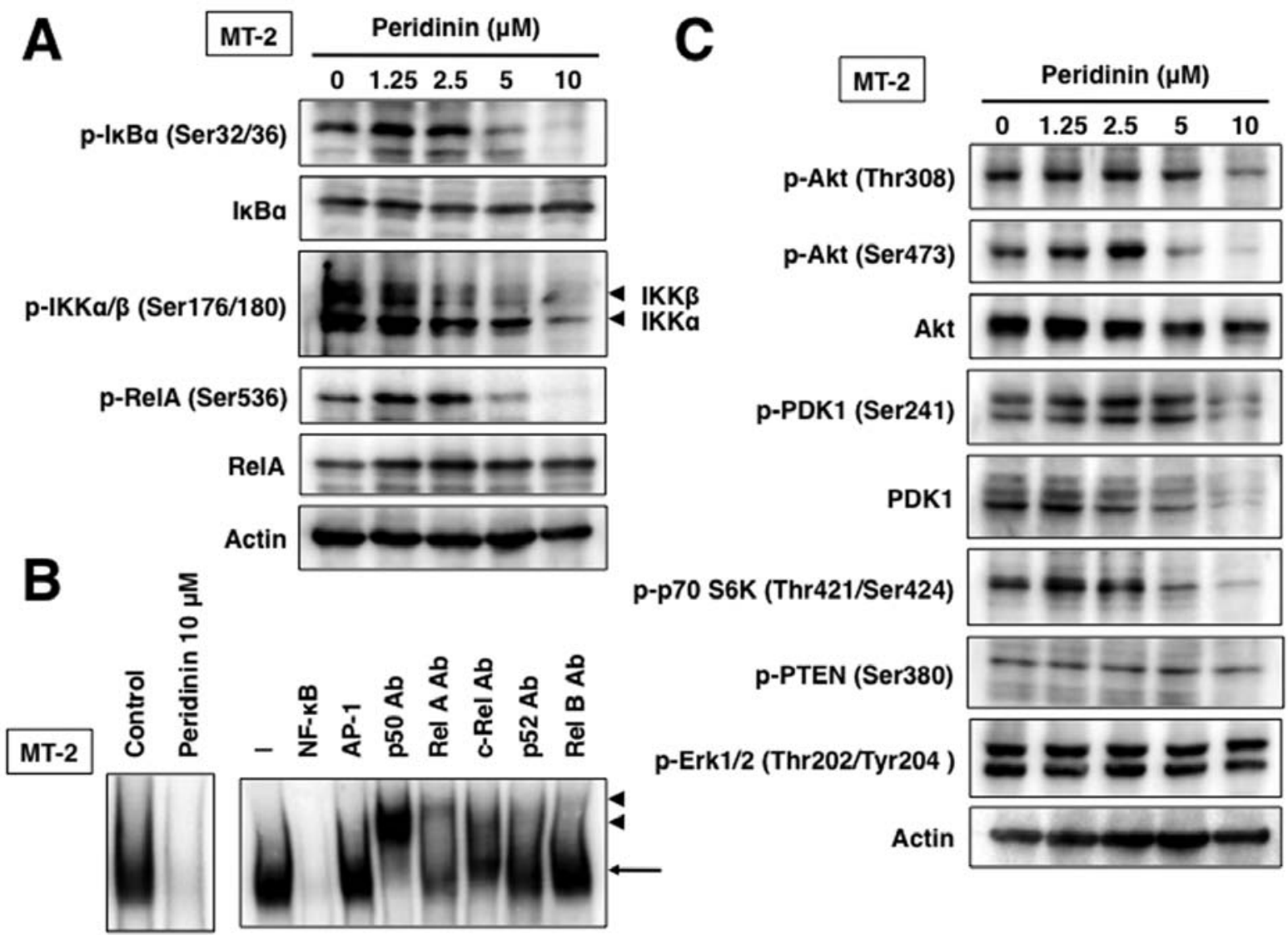

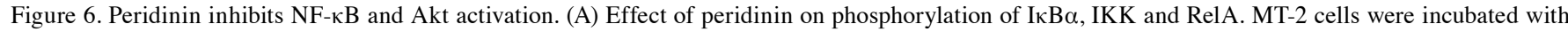
various concentrations of peridinin for $24 \mathrm{~h}$, and analyzed by western blot analysis using the indicated antibodies. (B) Effect of peridinin on DNA binding of

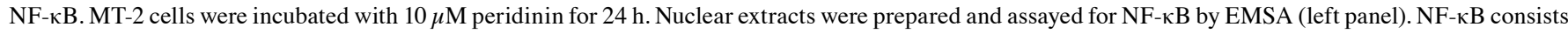
of p50, RelA, c-Rel, p52 and RelB subunits, and its binding to DNA is specific. Nuclear extracts were prepared from untreated MT-2 cells, incubated with

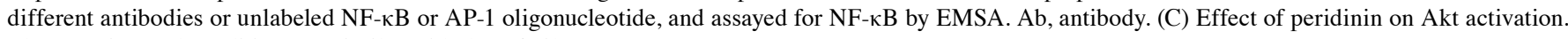
The experimental condition was similar with that of (A).

condensation, and nuclear fragmentation (Fig. 7E, left panel). TUNEL staining also demonstrated the presence of abundant apoptotic cells in the tumors of the peridinin-treated group (Fig. 7E, right panel). These findings suggest that the observed inhibition of tumor growth in the peridinin-treated tumors in vivo was mainly due to increased apoptosis.

Finally, to validate the results of the in vivo xenograft model, the effect of peridinin on the surrogate tumor markers sIL-2R (34) and sCD30 (35) by ELISA was investigated in serum samples. Compared with the control group, treatment of mice with peridinin significantly reduced serum sIL-2R and sCD30 levels (Fig. 7F).

\section{Discussion}

Our study showed that peridinin reduces cell proliferation and viability of HTLV-1-infected T-cell lines. These properties are similar to those described previously for fucoxanthin (5). Both carotenoids have similar structures, however, fucoxanthin is a $\mathrm{C}_{40}$-skeletal carotenoid with an octaenone chromophore whereas peridinin is a $\mathrm{C}_{37}$-skeletal butenolide carotenoid (Fig. 1). These results suggest no differences in the anti-ATL activities between butenolide ring-structure and octaenone chromophore carotenoids.
The goal of this study was to determine the effects of peridinin on ATL and the mechanism of action. Peridinin potently suppressed NF- $\kappa \mathrm{B}$ activation and also downregulated $\mathrm{NF}-\kappa \mathrm{B}-$ regulated gene products. The results also showed that peridinin inhibited $\mathrm{NF}-\kappa \mathrm{B}$ activation by suppressing IKK activation, and the latter was induced by suppressing Akt activation. In fact, Akt has been reported to activate IKK (26). While our results indicate that peridinin seems to inhibit IKK activation through the suppression of Akt activation, we cannot rule out direct inhibition of IKK activity by peridinin.

Our results also showed suppression of RelA phosphorylation by peridinin. Both IKK and Akt are involved in RelA phosphorylation (26). Thus, peridinin-induced inhibition of RelA phosphorylation could be mediated through the inhibition of both Akt and IKK. We also investigated the effect of peridinin on the upstream event of Akt, PDK1 and PTEN, and the results showed that peridinin inhibited the phosphorylation of Akt at Thr308 through PDK1 repression, but did not affect PTEN activity.

Our results showed that peridinin downregulated the expression of $\mathrm{NF}-\kappa \mathrm{B}$-regulated gene products involved in cellular proliferation (cyclin D1, cyclin D2, CDK4, CDK6 and $\mathrm{c}-\mathrm{Myc}$ ) and anti-apoptosis (XIAP, Bcl-2 and survivin). Akt also regulates cell cycle and proliferation by indirectly 

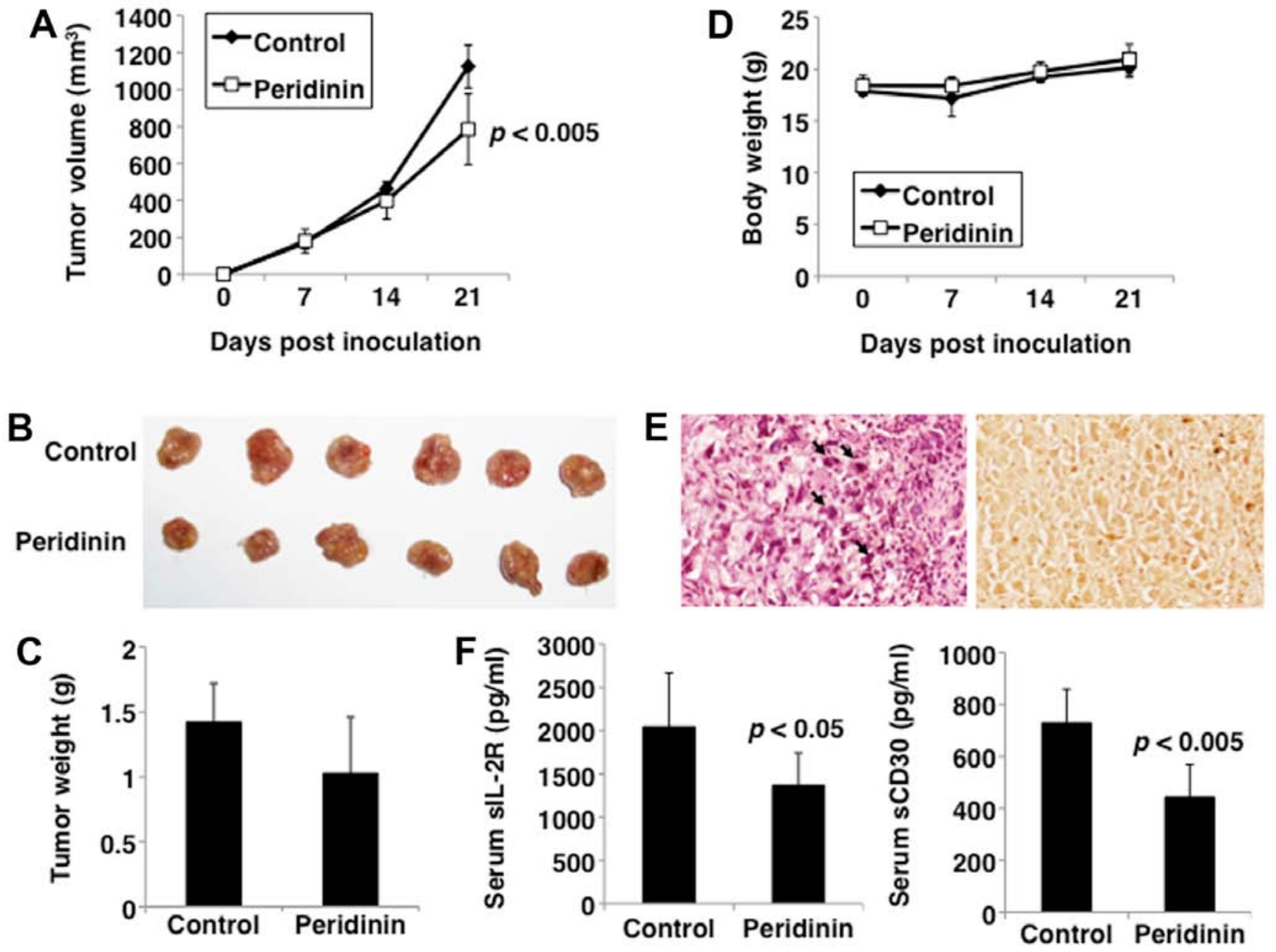

Figure 7. Antitumor efficacy and tolerability of peridinin in the HUT-102 ATL xenograft model. Tumor volume (A) and body weight (D) were monitored at regular intervals. Following 3 weeks of treatment, all SCID mice were sacrificed, and each tumor was excised and weighed. (B) Images of subcutaneously formed HUT-102 tumors and their weight (C) excised from mice of the control group and peridinin-treated group. (E) H\&E staining (left panel) and TUNEL expression (right panel) of the peridinin-treated group. Arrows show apoptotic cells. Magnification, x800. (F) Serum samples from peridinin-treated and untreated mice bearing HUT-102 tumors were assayed for sIL-2R (left panel) and sCD30 (right panel) by ELISA. Data are mean \pm SD of 6 mice/group.

modulating the levels of cyclin D1 and cyclin D2 (36). Akt interacts with and phosphorylates XIAP, a direct inhibitor of caspase-3 and caspase-9, leading to inhibition of ubiquitination and degradation of XIAP (37). In addition, XIAP is known to prevent apoptosis through upregulation of Akt cell survival signaling pathway (38). In addition, survivin and Bcl-2 have been shown to be downstream targets of Akt signaling $(39,40)$. Thus, NF- $\mathrm{KB}$ and Akt can collaborate in ATL, depending on various survival factors. The results indicate that peridinin downregulates $\mathrm{NF}-\kappa \mathrm{B}$-regulated gene products involved in cell proliferation and cell survival through inactivation of IKK and Akt.

Our in vivo experiments demonstrated that at a dose of $8.5 \mathrm{mg} / \mathrm{kg}$ body weight, peridinin markedly attenuated the growth of subcutaneous ATL xenografts. Notably, examination of tumors harvested from peridinin-treated mice showed increased apoptosis. The results also showed that at that dose, the treated mice showed no toxicity or any overt signs of ill health. These results highlight the anti-ATL and safety properties of peridinin. Further studies are needed to determine the long-term effects and safety of peridinin against experimentally-induced ATL.
In conclusion, the anti-ATL activity of peridinin was confirmed both in vitro and in vivo. Future studies should focus on the efficacy of peridinin and characterize its therapeutic potential against other human malignancies.

\section{Acknowledgements}

The authors thank Dr Taro Uchida for the excellent assistance. We also thank Dr Michiyuki Maeda for providing ED-40515(-) and the Fujisaki Cell Center, Hayashibara Biochemical Laboratories, Inc. (Okayama, Japan) for providing C5/MJ, HUT-102 and MT-1. This study was supported in part by JSPS KAKENHI grant numbers 15K18414 and 25461428.

\section{References}

1. Kannian P and Green PL: Human T lymphotropic virus type 1 (HTLV-1): Molecular biology and oncogenesis. Viruses 2: 2037-2077, 2010.

2. Rokkaku T, Kimura R, Ishikawa C, Yasumoto T, Senba M, Kanaya $\mathrm{F}$ and Mori N: Anticancer effects of marine carotenoids, fucoxanthin and its deacetylated product, fucoxanthinol, on osteosarcoma. Int J Oncol 43: 1176-1186, 2013. 
3. Tafuku S, Ishikawa C, Yasumoto T and Mori N: Anti-neoplastic effects of fucoxanthin and its deacetylated product, fucoxanthinol, on Burkitt's and Hodgkin's lymphoma cells. Oncol Rep 28: 1512-1518, 2012

4. Yamamoto K, Ishikawa C, Katano H, Yasumoto T and Mori N Fucoxanthin and its deacetylated product, fucoxanthinol, induce apoptosis of primary effusion lymphomas. Cancer Lett 300 : 225-234, 2011.

5. Ishikawa C, Tafuku S, Kadekaru T, Sawada S, Tomita M, Okudaira T, Nakazato T, Toda T, Uchihara JN, Taira N, et al: Anti-adult T-cell leukemia effects of brown algae fucoxanthin and its deacetylated product, fucoxanthinol. Int J Cancer 123 2702-2712, 2008.

6. Sugawara T, Yamashita K, Sakai S, Asai A, Nagao A, Shiraishi T, Imai I and Hirata T: Induction of apoptosis in DLD-1 human colon cancer cells by peridinin isolated from the dinoflagellate, Heterocapsa triquetra. Biosci Biotechnol Biochem 71: 1069-1072, 2007.

7. Haugan JA, Englert G, Aakermann T, Glinz E, Liaaen-Jensen S, Balzarini J, Fransson B, Ragnarsson U and Francis GW: Algal carotenoids 58. Isomerization studies on peridinin. Acta Chem Scand 48: 769-779, 1994

8. Ishiyama M, Miyazono Y, Sasamoto K, Ohkura Y and Ueno K A highly water-soluble disulfonated tetrazolium salt as a chromogenic indicator for NADH as well as cell viability. Talanta 44 $1299-1305,1997$

9. Zhang C, Ao Z, Seth A and Schlossman SF: A mitochondrial membrane protein defined by a novel monoclonal antibody is preferentially detected in apoptotic cells. J Immunol 157: 3980-3987, 1996

10. Mori $\mathrm{N}$ and Prager D: Transactivation of the interleukin-1alpha promoter by human T-cell leukemia virus type I and type II Tax proteins. Blood 87: 3410-3417, 1996.

11. Besbes S, Mirshahi M, Pocard M and Billard C: New dimension in therapeutic targeting of BCL-2 family proteins. Oncotarget 6: 12862-12871, 2015.

12. Li G, Chang H, Zhai YP and Xu W: Targeted silencing of inhibitors of apoptosis proteins with siRNAs: A potential anti-cancer strategy for hepatocellular carcinoma. Asian Pac J Cancer Prev 14: 4943-4952, 2013.

13. Malumbres M and Barbacid M: Cell cycle, CDKs and cancer: A changing paradigm. Nat Rev Cancer 9: 153-166, 2009.

14. Dang CV: c-Myc target genes involved in cell growth, apoptosis, and metabolism. Mol Cell Biol 19: 1-11, 1999.

15. Dang CV: $M Y C$ on the path to cancer. Cell 149: 22-35, 2012.

16. Pajic A, Spitkovsky D, Christoph B, Kempkes B, Schuhmacher M Staege MS, Brielmeier M,Ellwart J, Kohlhuber F, Bornkamm GW, et al: Cell cycle activation by c-myc in a Burkitt lymphoma model cell line. Int J Cancer 87: 787-793, 2000.

17. Zhou J, Ching YQ and Chng WJ: Aberrant nuclear factor-kappa B activity in acute myeloid leukemia: From molecular pathogenesis to therapeutic target. Oncotarget 6: 5490-5500, 2015.

18. Iwanaga R, Ohtani K, Hayashi T and Nakamura M: Molecular mechanism of cell cycle progression induced by the oncogene product Tax of human T-cell leukemia virus type I. Oncogene 20 : 2055-2067, 2001

19. Huang $Y$, Ohtani K, Iwanaga R, Matsumura $Y$ and Nakamura $M$ Direct trans-activation of the human cyclin D2 gene by the oncogene product Tax of human T-cell leukemia virus type I. Oncogene 20: 1094-1102, 2001.

20. Iwanaga R, Ozono E, Fujisawa J, Ikeda MA, Okamura N, Huang Y and Ohtani K: Activation of the cyclin D2 and cdk6 genes through NF-kappaB is critical for cell-cycle progression induced by HTLV-I Tax. Oncogene 27: 5635-5642, 2008

21. Duyao MP, Kessler DJ, Spicer DB, Bartholomew C, Cleveland JL, Siekevitz M and Sonenshein GE: Transactivation of the c-myc promoter by human $\mathrm{T}$ cell leukemia virus type 1 tax is mediated by NF $\kappa B$. J Biol Chem 267: 16288-16291, 1992.

22. Kawakami A, Nakashima T, Sakai H, Urayama S, Yamasaki S, Hida A, Tsuboi M, Nakamura $\mathrm{H}$, Ida H, Migita $\mathrm{K}$, et al: Inhibition of caspase cascade by HTLV-I tax through induction of NF-kappaB nuclear translocation. Blood 94: 3847-3854, 1999.
23. Akita K, Kawata S and Shimotohno K: p2 ${ }^{\mathrm{WAF} 1}$ modulates NF-kappaB signaling and induces anti-apoptotic protein Bcl-2 in Tax-expressing rat fibroblast. Virology 332: 249-257, 2005.

24. Kawakami H, Tomita M, Matsuda T, Ohta T, Tanaka Y, Fujii M, Hatano M, Tokuhisa $\mathrm{T}$ and Mori N: Transcriptional activation of survivin through the NF-kappaB pathway by human T-cell leukemia virus type I tax. Int J Cancer 115: 967-974, 2005.

25. Hinz M, Krappmann D, Eichten A, Heder A, Scheidereit C and Strauss M: NF-kappaB function in growth control: Regulation of cyclin D1 expression and G0/G1-to-S-phase transition. Mol Cell Biol 19: 2690-2698, 1999.

26. Viatour P, Merville MP, Bours V and Chariot A: Phosphorylation of NF-kappaB and IkappaB proteins: Implications in cancer and inflammation. Trends Biochem Sci 30: 43-52, 2005.

27. Casamayor A, Morrice NA and Alessi DR: Phosphorylation of Ser-241 is essential for the activity of 3-phosphoinositidedependent protein kinase-1: Identification of five sites of phosphorylation in vivo. Biochem J 342: 287-292, 1999.

28. Alessi DR, James SR, Downes CP, Holmes AB, Gaffney PRJ, Reese CB and Cohen P: Characterization of a 3-phosphoinositidedependent protein kinase which phosphorylates and activates protein kinase Balpha. Curr Biol 7: 261-269, 1997.

29. Pullen N, Dennis PB, Andjelkovic M, Dufner A, Kozma SC, Hemmings BA and Thomas G: Phosphorylation and activation of p70 $0^{\mathrm{s} 6 \mathrm{k}}$ by PDK1. Science 279: 707-710, 1998.

30. Hollander MC, Blumenthal GM and Dennis PA: PTEN loss in the continuum of common cancers, rare syndromes and mouse models. Nat Rev Cancer 11: 289-301, 2011.

31. Nakahata S, Ichikawa T,Maneesaay P, Saito Y,Nagai K, Tamura T, Manachai N, Yamakawa N, Hamasaki M, Kitabayashi I, et al: Loss of NDRG2 expression activates PI3K-AKT signalling via PTEN phosphorylation in ATLL and other cancers. Nat Commun 5: 3393, 2014.

32. Rahdar M, Inoue $T$, Meyer $T$, Zhang J, Vazquez $F$ and Devreotes PN: A phosphorylation-dependent intramolecular interaction regulates the membrane association and activity of the tumor suppressor PTEN. Proc Natl Acad Sci USA 106: 480-485, 2009

33. Stoppa G, Rumiato E and Saggioro D: Ras signaling contributes to survival of human T-cell leukemia/lymphoma virus type 1 (HTLV-1) Tax-positive T-cells. Apoptosis 17: 219-228, 2012.

34. Kamihira S, Atogami S, Sohda H, Momita S, Yamada Y and Tomonaga M: Significance of soluble interleukin-2 receptor levels for evaluation of the progression of adult T-cell leukemia. Cancer 73: 2753-2758, 1994.

35. Nishioka C, Takemoto S, Kataoka S, Yamanaka S, Moriki T, Shoda M, Watanabe T and Taguchi H: Serum level of soluble CD30 correlates with the aggressiveness of adult T-cell leukemia/ lymphoma. Cancer Sci 96: 810-815, 2005.

36. Zhang X, Tang N, Hadden TJ and Rishi AK: Akt, FoxO and regulation of apoptosis. Biochim Biophys Acta 1813: 1978-1986, 2011

37. Dan HC, Sun M, Kaneko S, Feldman RI, Nicosia SV, Wang HG, Tsang BK and Cheng JQ: Akt phosphorylation and stabilization of X-linked inhibitor of apoptosis protein (XIAP). J Biol Chem 279: 5405-5412, 2004

38. Cheng JQ, Jiang X, Fraser M, Li M, Dan HC, Sun M and Tsang BK: Role of X-linked inhibitor of apoptosis protein in chemoresistance in ovarian cancer: Possible involvement of the phosphoinositide-3 kinase/Akt pathway. Drug Resist Updat 5: 131-146, 2002.

39. Liang Y-L, Wang L-Y, Wu H, Ma D-Z, Xu Z and Zha X-L: PKB phosphorylation and survivin expression are cooperatively regulated by disruption of microfilament cytoskeleton. Mol Cell Biochem 254: 257-263, 2003

40. Pugazhenthi S, Nesterova A, Sable C, Heidenreich KA, Boxer LM, Heasley LE and Reusch JE-B: Akt/protein kinase B up-regulates Bcl-2 expression through cAMP-response elementbinding protein. J Biol Chem 275: 10761-10766, 2000. 